\title{
Measuring visual neglect in acute stroke and predicting its recovery: the visual neglect recovery index
}

\author{
SP Stone, P Patel, RJ Greenwood, PW Halligan
}

\begin{abstract}
An overall measure of the recovery of visual neglect in patients with an acute stroke is described: The "Visual Neglect Recovery Index" (VNRI) expresses the amount of visual neglect on a battery of visual neglect tests as a percentage of complete recovery from the maximal visual neglect measurable. The principles underlying the development of the index are similar to those involved in the development of the Motricity Index for hemiplegia. A population of 68 survivors of stroke who presented with visual neglect at two to three days were followed for up to six months. The VNRI showed that neglect was greater in those with right hemisphere stroke than in those with left hemisphere stroke and that recovery was most rapid over the first 10 days and reached a plateau at three months. Most patients, including many with severe initial visual neglect, showed little visual neglect at three months. Stepwise regression analysis showed that the severity of visual neglect at three months and at six months post-stroke could be predicted by the severity of visual neglect and the presence of anosognosia at two to three days. A regression equation was produced which may enable clinicians to select patients for intensive treatment of visual neglect.
\end{abstract}

Department of Neurosciences, St Bartholomew's Hospital, London SP Stone

RJ Greenwood

Department of Computer Studies, St Bartholomew's Hospital, London P Patel

Rivermead

Rehabilitation Centre and Neuropsychology Unit, University of Oxford

PW Halligan

Correspondence to: Dr Stone,

Academic Department, Geriatric Medicine, Royal Free Hospital, Pond Street, London NW3 2QG, UK

Received 11 December 1991

and in revised form

13 August 1991.

Accepted 22 August

1991
Visual neglect may recover in some patients with an acute stroke ${ }^{12}$ but for some it represents a serious rehabilitation problem..$^{3-5}$ At present, opinion is divided as to the effectiveness of neuropsychological treatments for visual neglect. ${ }^{6-9}$ To date, no trial has randomised patients according to prognosis because the features that predict recovery are unknown. Knowledge of these are essential to select comparable groups of patients for controlled trials of treatment. Only then can the best use be made of intensive rehabilitation resources.

Some of the reasons why the predictive features are unknown are because previous studies have examined patients at different times post-stroke, have not used clear definitions of neglect phenomena and have used different tests to detect visual neglect. ${ }^{10}$ We have recently developed a standardised test battery for the assessment of visual neglect and acute stroke. ${ }^{1011}$ We report the findings of a study in which patients with visual neglect at two to three days were followed for up to six other neglect phenomena in patients with an months post stroke. To express performance on the tests of the battery as a single overall figure, a visual neglect index was developed, which was then used to predict the severity of visual neglect at three and six months post-stroke.

The test battery is a modified form of the Behavioural Inattention Test. ${ }^{10-12}$ Performance on each test of the battery is expressed as a grade on a $0-5$ scale that reflects the percentage of items omitted on each test (grade 0: no neglect; grade 1: $0-20 \%$; grade 2: $21-40 \%$; grade $3: 41-60 \%$; grade $4: 61-80 \%$; grade 5 : 81-100\%). Although, this grading system provides a profile of scores and is sensitive to change $^{10}$ it is not as useful as a single overall score. The simplest solution would appear to be to summate and average the graded scores on the entire battery. This, however, cannot be done for two reasons. First, these grades are in arbitrary units of no real value, so they cannot be treated as continuous variables. Second, some patients are unable to do all the tests because of aphasia or drowsiness. Since it cannot be assumed that each test measures the same neuropsychological variable, ${ }^{13-15}$ grade 3 neglect on one test may not be equivalent to grade 3 neglect on another.

The problem is similar to that encountered when trying to express the power loss after stroke as an overall score. Demeurisse et al ${ }^{16}$ overcame this problem by developing the Motricity Index. This converted the arbitrary 0-5 grades of the MRC scale for muscle movements at individual joints into a single overall figure expressing the power as a percentage of "total motor recovery"; $0 \%$ represented no recovery and $100 \%$ full recovery from total hemiplegia. The principles underlying the development of the motricity index were used to create an overall measure of visual neglect recovery, the "Visual Neglect Recovery Index" (VNRI), from the graded performance of patients on the individual tests in the neglect test battery. This measure was then used in predicting recovery of visual neglect.

\section{Method}

One hundred and seventy one consecutive patients with an acute hemispheric first stroke were examined for evidence of visual neglect at two to three days post-stroke using the neglect test battery as described. ${ }^{10}$ The presence of the following neglect phenomena and related disorders was also assessed using standardised measures ${ }^{11}$ and was recorded as present (1) or absent (0): behavioural neglect, visual extinction, sensory extinction, allaesthesia, anosog- 
nosia ${ }^{17}$ visual field defect and gaze paresis. The patients age was noted. The severity of power loss was assessed using the Motricity Index. ${ }^{16}$ The level of consciousness was recorded as unimpaired (0), drowsy or comatose (1). ${ }^{18}$

A group of 68 patients ( 34 right hemisphere and 34 left hemisphere) presenting with visual neglect, who survived at least three months and whose subsequent course was unaffected by other disease or a second stroke formed the study population. These patients were reexamined at 10 days, three weeks, six weeks and three months. Patients were seen again at six months but six were lost to further follow up, one died of other disease, one died of the late complications of stroke induced immobility and one suffered a second stroke. Follow up was discontinued when visual neglect appeared to have resolved.

Each patient's graded score on each test of the battery at three days and at three months was recorded. Three months was chosen as the end point because the majority of patients make most of their recovery at three months. ${ }^{11920}$ If neglect had resolved in a patient at three weeks it was assumed that no visual neglect would have been present at three months.

The battery includes six tests which are done by both right and left hemisphere stroke patients: indicating items of food on a plate (meal), reading a menu (menu), selecting named coins from an array on a card (coins), pointing to objects scattered round the room (pointing), cancelling lines on a piece of paper (lines) and cancelling stars (stars). Performance on each was graded $0-5$, as above. ${ }^{10}$

For each test a double entry table was constructed, such as that for Reading the Menu (table 1). The figures in the first vertical column refer to the number of patients who had grade $0,1,2,3,4$, or 5 neglect on the Menu at three days post-stroke who also had grade 0 on that test at three months. The second vertical column gives the number of patients whose initial grade was $0,1,2,3,4$ or 5 and whose final grade at three months was grade 1, and so on. Using the method described for weighting the stages in the Motricity Index, ${ }^{16}$ the difficulty experienced by individual patients in progressing, in Menu, from one grade to the next over the first three months was compared with the total difficulty experi-
Table 1 Double entry table for Reading a Menu

\begin{tabular}{|c|c|c|c|c|c|c|c|}
\hline \multirow[t]{2}{*}{. } & & \multicolumn{6}{|c|}{ Grade neglect at 3 months } \\
\hline & & 0 & 1 & 2 & 3 & 4 & 5 \\
\hline \multirow[t]{6}{*}{ Grade neglect at 3 days } & 0 & 8 & & & & & \\
\hline & 1 & 1 & 0 & & & & \\
\hline & 2 & 3 & 1 & 0 & & & \\
\hline & 3 & 12 & 1 & 0 & 0 & & \\
\hline & 4 & 7 & 1 & 2 & 1 & 0 & \\
\hline & 5 & 3 & 0 & 0 & 2 & 2 & 1 \\
\hline
\end{tabular}

enced in progressing from maximum neglect (grade 5) to no neglect (grade 0), over that period (Appendix). This relative difficulty was expressed as a percentage so that, for example, change from grade 3 to 2 neglect on Menu was equivalent to progress from $46-67 \%$ of total neglect recovery.

Applying this method to all tests enabled the percentage recovery to be calculated for each grade on each test. The percentage score on each test could be summated and averaged for each patient's performance over the entire battery because the measurements on each test were now in equivalent units. This gave an overall measure of visual neglect recovery which was called the "Visual Neglect Recovery Index" (VNRI). A patient with maximum visual neglect on the battery had a VNRI score of $0 \%$ while a patient with no discernable visual neglect had a VNRI score of $100 \%$.

The correlation of the VNRI with the percentage recovery score on individual tests at three days was assessed by analysis of variance and by least significant difference analysis. The change in the mean VNRI values with time was examined for all 68 patients and for right and left hemisphere stroke patients separately.

Stepwise regression analysis was carried out using the BMDP statistical package. ${ }^{21}$ The independent (predictive) variables were the clinical assessments carried out at two to three days post-stroke and the dependent variables (outcomes) were the severity of visual neglect at three months and at six months. Outcome at three months was chosen for reasons explained above and the six month outcome was chosen because up to $30 \%$ of patients may make considerable gains in specified areas of dysfunction between three and six months. ${ }^{19}$

Table 2 Value of each grade on each test as a percentage of total visual neglect recovery on that test over three months

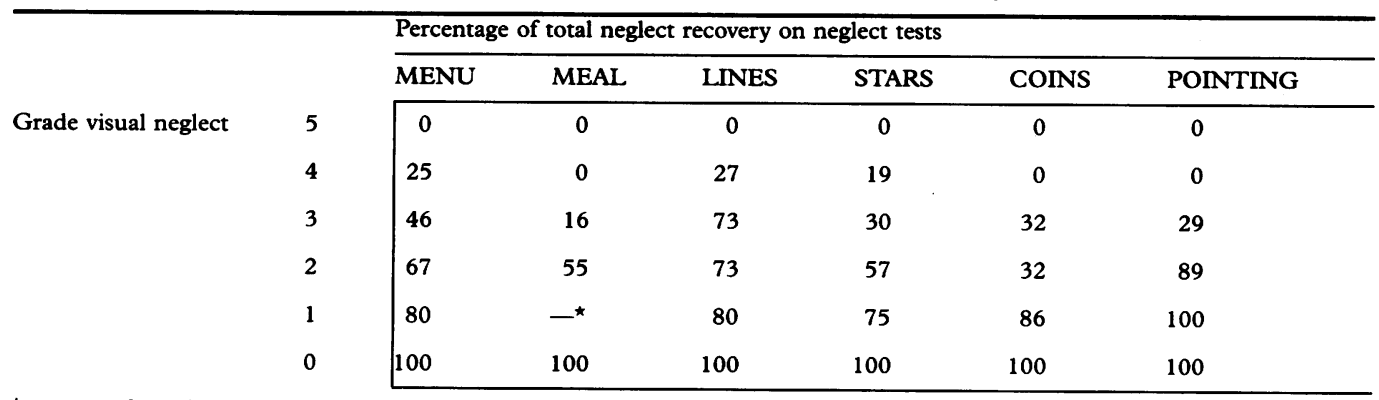

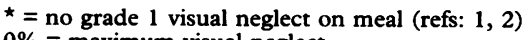

$0 \%=$ maximum visual neglect

$100 \%=$ no visual neglect 


\section{Results}

1) Percentage recovery represented by each grade on each test

The percentage recovery represented by each grade on each of the six tests is given in table 2 . This table, for example, suggests that it is just as difficult to recover from grade 2 as grade 3 neglect on Line cancellation; that it is more difficult to recover from grade 3 neglect on Meal than from grade 3 on Line cancellation.

The table was used to summate and average a patient's percentage recovery score on each test to give an overall measure of visual neglect. For example, a patient with grade 2 neglect on Meal ( $55 \%$ recovery), grade 3 on Menu ( $46 \%$ recovery), and grade 0 ( $100 \%$ recovery) on the other tests at six weeks post-stroke, has an average recovery score (VNRI) of $83.5 \%$.

If a patient was able to attempt only three or four tests because of aphasia or tiredness, the VNRI was calculated from the results of those tests as measurements were now in equivalent non-arbitrary units of "percentage recovery".

\section{2) Change in VNRI over time}

Figure 1 shows a plot of the mean VNRI at different time intervals up to six months for right and left hemisphere patients taken separately.

Visual neglect recovers most quickly over the first 10 days and reaches a plateau at three months. Recovery is significantly greater in those with a left hemisphere stroke than in those with a right hemisphere stroke at three

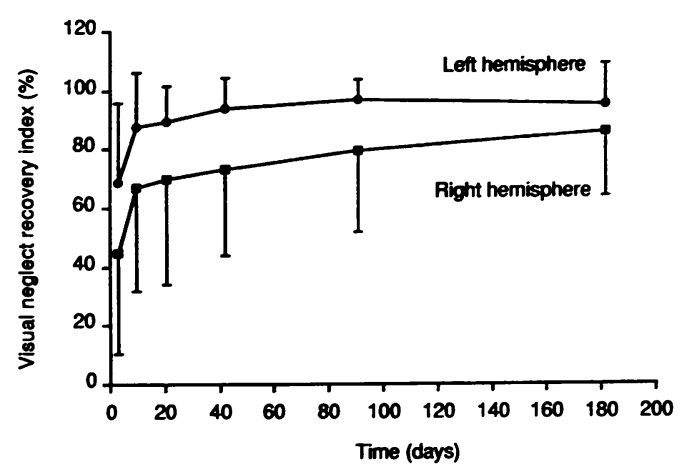

Figure 1 Change in visual neglect recovery index over time in patients with right $(n=34)$ and left $(n=34)$ hemisphere stroke.

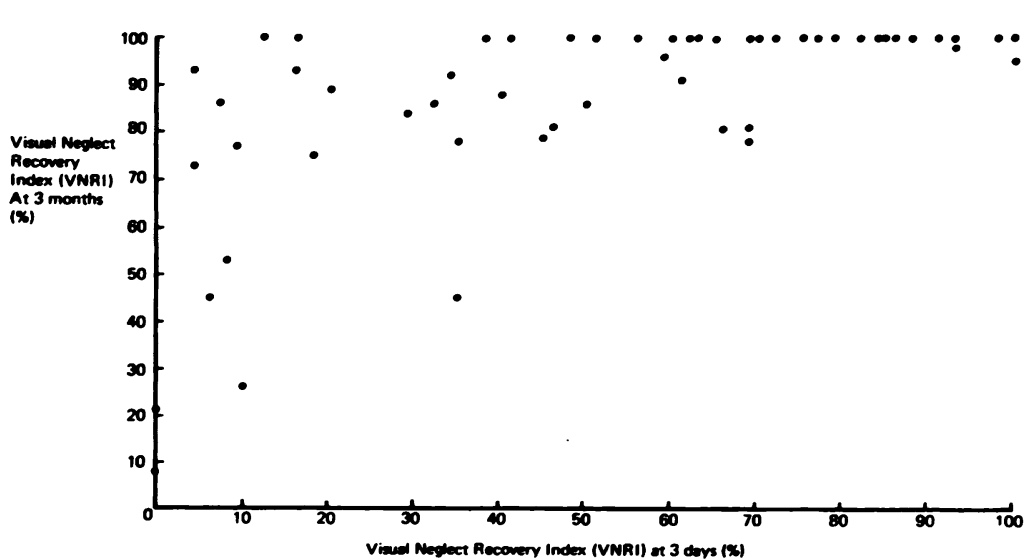

Figure 2 Visual neglect recovery index in stroke patients at 3 days and at 3 months post-stroke. days $(t=3.23 ; \mathrm{p}<0.025 ; \mathrm{n}=68), 10$ days $(\mathrm{t}=2.89 ; \quad \mathrm{p}<0.05 ; \mathrm{n}=64)$, three weeks $(t=2.96 ; \quad p<0.025 ; \quad n=66)$, six weeks $(\mathrm{t}=3.7 ; \mathrm{p}<0.005 ; \mathrm{n}=66)$ and three months $(t=3.55 ; p<0.005 ; n=68)$, but not at six months $(t=1.94 ; p=0.4 ; n=62)$.

The recovery between three days and 10 days is significant for right hemisphere $(t=5.77 ; \mathrm{df}=32 ; \mathrm{p}<0.002)$ and for left hemisphere strokes $(t=5.43 ; \quad d f=30$; $p<0.002)$. Recovery between 10 days and three months is significant for both groups (right hemisphere: $\mathrm{t}=3.33$; $\mathrm{df}=32 ; \mathrm{p}<0.01$; Left hemisphere: $t=3.72 ; \quad d f=30$; $p<0.002)$. The change from three to six months is significant for right hemisphere $(t=2.57 ; \mathrm{df}=27 ; \mathrm{p}=0.04)$ but not for left hemisphere stroke $(t=0.93 ; \mathrm{df}=33 ;$ N.S. $)$.

Many patients have little or no residual visual neglect at three months. This is true even of the 15 patients presenting with a VNRI of 0-20\%, seven of whom attained a three month score of at least $75 \%$ (figure 2 ). In the whole population only seven patients had a three month VNRI of below $60 \%$; all, except one, had a VNRI of at least $75 \%$.

\section{3) Correlation of VNRI with individual test scores}

Change over time in the individual test scores, taken for all 68 patients, follows a similar pattern to that for the VNRI as a whole (figure 3). Star cancellation recovers least. At all stages post-stroke, there was a general trend showing that as recovery in each test increases so does recovery over the whole battery as reflected in the VNRI, although detailed examination shows wide variation between individuals. Calculation of correlation coefficients is not valid because the individual test scores fall into categorical groups. Analysis of variance is difficult because some groups are very small. However, where it was possible ANOVA showed that at three days there was a significant difference between the VNRI means for each level of visual neglect on Meal ( $F=59.27$ at $3,46 \mathrm{df}$ ) and on Pointing ( $F=27 \cdot 13$, at $3,45 \mathrm{df}$ ). This was confirmed by Least Significant Difference analysis.

4) Prediction of the severity of visual neglect at three and six months

At both time points two variables were found to make an individual independent contribu-

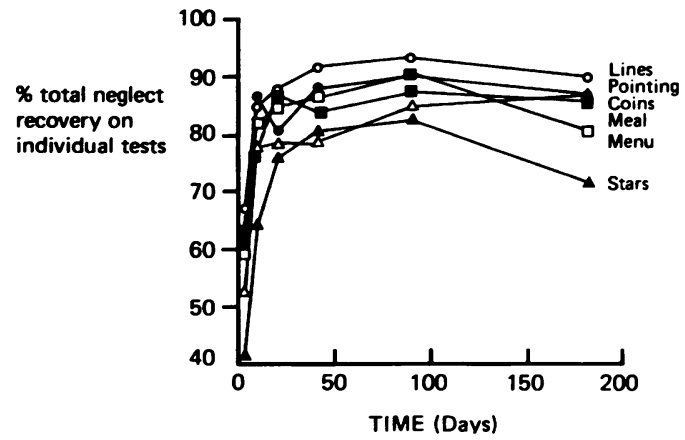

Figure 3 Change in individual neglect test scores with time. 


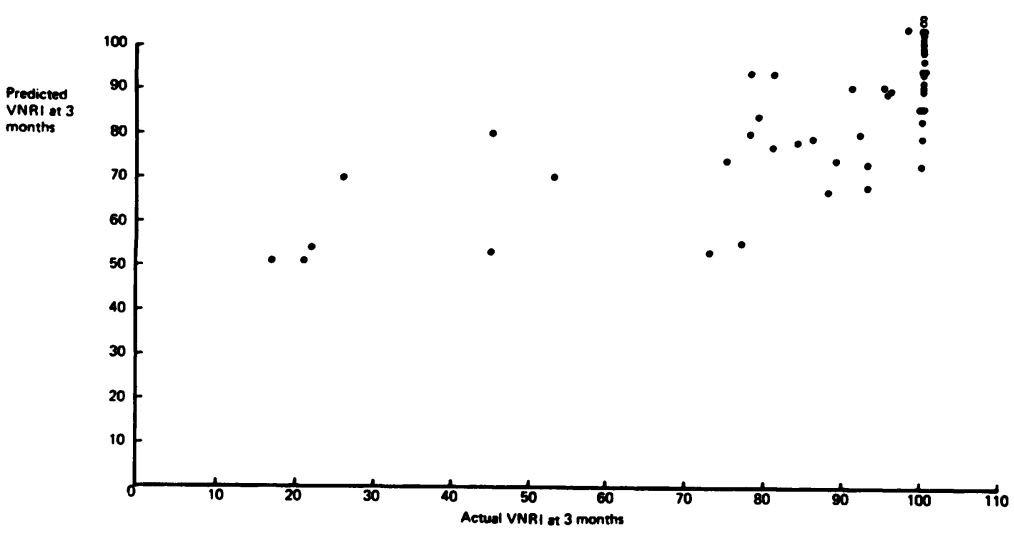

Figure 4 Comparison of actual and predicted VNRI scores at 3 months.

tion to outcome. These were the severity of visual neglect and the presence of anosognosia at two to three days post-stroke. The following regression equations were derived which relate the severity of visual neglect at both time points to the significant predictor variables:

VNRI ( 3 months) $=67+0.4$ (VNRI 3 days) $-15 \times$ Anosognosia.

VNRI (6 months) $=74.5+0.3$ (VNRI 3 days) $-12 \times$ Anosognosia.

The equation for prediction at three months was based on 55 cases while that for six months was based on 49 cases. The independent variables explained $53 \%$ of the variance at three months and $46 \%$ at six months. The similarity of the equations demonstrated the consistency of the independent variables in predicting outcome.

Figure 4 shows how the actual and predicted VNRI scores at three months compare. Predicted and actual scores match well at the upper end of the VNRI. At the lowest end of the range, matching is poor, with frequent over-estimation of recovery. This was true at six months as well.

Residuals were plotted against the predicted values and also against the variables that were not in the model. Residual analysis showed a good scatter of results and confirmed that there were no other significant predictors of outcome.

\section{Discussion}

The VNRI expresses a patient's performance on a standardised battery of tests as a percentage of complete recovery from the maximum visual neglect measurable. It enables clinicians to express the severity of visual neglect as a single figure and may help predict outcome. It is a better option than summating and averaging the graded scores of individual tests because the VNRI expresses each grade in equivalent units of "neglect recovery" instead of arbitrary units of no real value. Visual neglect may be task-specific ${ }^{13}$ and the VNRI may facilitate comparison between performance on different tests. There is reasonable correlation between individual test scores and the VNRI, suggesting that if a patient is unable to do one or two tests because of aphasia or drowsiness, the VNRI derived from the tests done by the patient is probably representative of their visual neglect on the entire battery. It could be argued that one test could be as useful as the entire battery, but recent work has illustrated the differential sensitivity of individual tests ${ }^{15}$ and stressed that use of only one test might result in failure to detect a significant proportion of patients with neglect. ${ }^{223}$ The index, like the BIT $^{12}$ from which the test battery was derived, does not specifically lateralise the omissions, but in practice, only $2 \%$ of test results are not clearly lateralised.

The rate of recovery of visual neglect demonstrated by the VNRI is similar to that reported for other neurological deficits or disabilities in stroke ${ }^{2024}$ and by a much smaller study of visual neglect which used only one test. ${ }^{1}$ Recovery is slower for those with a right hemisphere stroke and continues between three and six months. The hemispheric difference in severity is consistent with findings of other studies. ${ }^{102526}$

The VNRI at two to three days and the presence or absence of anosognosia can be used to predict the degree of recovery of visual neglect. These predictor variables make clinical sense in that the initial severity of visual neglect affects its severity at three months and at six months. The influence of anosognosia on outcome is of particular interest. Patients with anosognosia had a significantly greater degree of visual neglect (mean VNRI 34\%,SD 38\%) than those without anosognosia (mean VNRI $62 \%$, SD 31\%; $t=12.87$, df 53, $p<0.0005$ ). The presence of anosognosia may therefore be regarded as an indicator of severe visual neglect, ${ }^{27}$ and hence, poor recovery, even though operational definitions of the two deficits have been shown to be clinically dissociable. ${ }^{1728}$ However, in this study, a few patients with anosognosia had little visual neglect.

The predictive equations derived are simple and are consistent for both time points. There was good matching of actual and predicted recovery at the upper range. At the lower range, however, prediction of the exact severity of visual neglect in the worst patients was less precise. This is likely to be because there were too few such patients at three months to allow more accurate stepwise regression analysis. Nonetheless, of the eight patients with a three month VNRI of $<75 \%$, seven were correctly predicted as such and it is from this group that candidates for intensive treatment of neglect might be expected to come. Some of the inaccuracies in prediction may also reflect the failure to standardise remedial therapy in the population studied; hence some patients may have received more treatment for visual neglect than others. The best test of the model's accuracy would be to carry out the prediction analysis on a new sample of patients. This would also demonstrate that the predictive power of the VNRI was not solely the result of the statistical tautology inherent in its development.

Nonetheless, it is apparent that in most patients visual neglect makes a good recovery. ${ }^{12}$ The predictive equations may be of use 
in randomising patients for trials of intensive treatment of visual neglect. For example, all but one of those whose VNRI at three months was $<75 \%$ had a predicted VNRI of $<75 \%$ and it might be from this group of patients that candidates for such treatment might come. The current study demonstrates the difficulty of recruiting sufficient patients for such a trial, given the generally good prognosis for the recovery of visual neglect.

SPS was supported by a grant from the Chest Heart and Stroke Association.

1 Wade DT, Wood VA, Hewer RL. Recovery of cognitive function soon after stroke; a study of visual neglect, attention span and verbal recall. $\mathcal{f}$ Neurol Neurosurg Psychiatry 1988;51:10-13.

2 Hier DB, Mondlock J, Caplan LR. Recovery of behavioural abnormalities after right hemisphere stroke. Neurology 1983;33:345-50.

3 Kinsella G, Ford B. Acute recovery patterns in stroke patients. Neuropsychological factors Med $f$ Australia 1980;2:663-6.

4 Denes G, Semenza C, Stoppa E, Lis A. Unilateral spatial neglect and recovery from hemiplegia. A follow-up study. Brain 1982;105:543-52.

5 Fullerton KJ, Mackenzie G, Stout RW. Prognostic indices in stroke. $Q \ngtr$ Med 1988;66:147-62.

6 Weinberg J, Diller L, Gordon WA, et al. Visual scanning training effect on reading related tasks in acquired righ brain damage. Arch Phys Med Rehabil 1977;58:479-86.

7 Weinberg J, Diller L, Gordon W, et al. Training sensory awareness and spatial organization in people with right brain damage. Arch Phys Med Rehabil 1979;60:491-6.

8 Gordon W, Hibbard MR, Egelko S, et al. Perceptual remediation in patients with right brain damage: remediation in patients with right brain damage: a
comprehensive program. Arch Phys Med Rehabil comprehensive

9 Robertson IH, Gray JM, Pentland B, Waite LJ. A randomized controlled trial of microcomputer-based rehabilitation for unilateral left visual neglect. Arch Phys Med Rehabil 1990;71:663-8.

10 Stone SP, Wilson B, Halligan $\mathrm{P}$, et al. The assessment of visual neglect in acute stroke. $\mathcal{f}$ Neurol Neurosurg Psychiatry 1991;54:345-50.

11 Stone SP, Halligan P, Wilson B, Marshall J, Greenwood RJ. Performance of age-matched controls on a battery of visuo-spatial neglect tests. I Neurol Neurosurg Psychiatry visuo-spatial negle

12 Wilson B, Cockburn J, Halligan P. The Behavioural Inattention Test. Fareham, Hampshire: Thames Valley Test Comtion Test. Fareh

13 Halsband U, Gruhn S, Ettlinger G. Unilateral spatial neglect and defective performance in one half of space. Intern $\mathcal{F}$ Neurosci 1985;28:173-95.

14 Bisiach E, Perani D, Vallar G, Berti A. Unilateral neglect: personal and extra-personal. Neuropsychologia 1986;24:759-67.

15 Halligan PW, Marshall JC, Wade DT. Visuospatial neglect: underlying factors and test sensitivity. Lance 1989;2:908-10.

16 Demeurisse G, Demol O, Robaye E. Motor evaluation in vascular hemiplegia. Eur Neurol 1980;19:382-9.

17 Cutting J. The study of anosognosia. $\mathcal{F}$ Neurol Neurosurg Psychiatry 1978;41:548-55.

18 Allen CMC. Predicting the outcome of acute stroke: a prognostic score. $f$ Neurol Neurosurg Psychiatry prognostic score.

19 Andrews K, Brocklehurst C, Richards B, Laycock PJ. The rate of recovery from stroke and its measurement. Int Rehab Med 1981;3:155-61.

20 Skilbeck CE, Wade DT, Hewer RL, Wood VA. Recovery after stroke. I Neurol Neurosurg Psychiatry 1983;46:5-8.

21 Dixon WJ. BMDP Statistical Software. University of California Press, California: 1985.

22 Ogden JA. Anterior-Posterior interhemispheric differences in the loci of lesions producing visual hemineglect. Brain Cognit 1985;4:59-75.

23 Stone SP, Greenwood RJ. Assessment of visual neglect. Lancet 1991;337:114 (letter).

24 Wade DT, Wood VA, Hewer Ri. Recovery after stroke: the first three months. $\mathcal{f}$ Neurol Neurosurg Psychiatry first three mo

25 Chedru F, Leblanc M, L'Hermitte F. Visual searching in normal and brain-damaged subjects (a contribution to

26 Chain F, Leblanc M, Chedru F, L'Hermitte F. Negligence visuelle dans les lesions posterieures de l'hemisphere gauche. Rev Neurol 1979;135:105-26.

27 Friedland RP, Weinstein EA. Hemi-inattention and hemisphere specialization: introduction and historical review. Advances in Neurology 1977;18:1-31.

28 Bisiach E, Vallar G, Perani D, Papgno C, Berti A. Unawareness of disease following lesions of the right hemisphere: anosognosia for hemiplegia and hemianopia. Neuropsychologia 1986;24:471-82.

\section{Appendix}

Table 1 shows that in Reading the Menu there were 8 patients who initially had grade 5 neglect on this test. Seven of these $(3+0+0$ $+2+2$ ) improved over 3 months. The percentage $\left(\mathrm{X}^{1}\right)$ of those who recovered from grade 5 to grade 4 at some stage over the 3 months is $7 / 8 \times 100 \%$, that is, $87.5 \%$. Similarly, the number of patients whose initial grade at 3 days was grade 4 or who had recovered from grade 5 to 4 at some stage over the 3 months is $7+1+2+1+0+3+0+0$ $+2+2=18$. Sixteen of these $(7+1+2+1$ $+3+0+0+2$ ) recovered from grade 4 to 3 at some time over the 3 months. The percentage $\left(\mathrm{X}^{2}\right)$ who recovered from grade 4 to 3 is, therefore, $16 / 18 \times 100 \%$ (that is, $89 \%$ ). Similar percentages $\mathrm{X}^{3}, \mathrm{X}^{4}, \mathrm{X}^{5}$ were calculated for the patients recovering from Grade 3 to 2, 2 to 1 , and 1 to 0 at some stage over 3 months.

If every patient had had grade 5 neglect at 3 days the percentage of those who would have recovered from grade 5 to 0 over 3 months would be

$$
\begin{array}{r}
\frac{X^{1}}{100} \times \frac{X^{2}}{100} \times \frac{X^{3}}{100} \times \frac{X^{4}}{100} \times \frac{X^{5}}{100} \times 100 \%= \\
\frac{X^{1} \cdot X^{2} \cdot X^{3} \cdot X^{4} \cdot X^{5}}{10^{8}}=58 \%
\end{array}
$$

The percentage of patients who did not recover from grade 5 to 4 is $100-\mathrm{X}^{1}$ from grade 4 to 3 is $100-X^{2}$ and so on. The percentage who would not have recovered from grade 5 to 0 can be given by

$$
\begin{aligned}
& 100-\frac{X^{1} \cdot X^{2} \cdot X^{3} \cdot X^{4} \cdot X^{5}}{10^{8}}= \\
& \frac{10^{10}-X^{1} \cdot X^{2} \cdot X^{3} \cdot X^{4} \cdot X^{5}}{10^{8}}=42 \%
\end{aligned}
$$

The coefficients $\mathrm{D}^{1}, \mathrm{D}^{2}, \mathrm{D}^{3}, \mathrm{D}^{4}, \mathrm{D}^{5}$, comparing the relative difficulty in recovering from grade 5 to 4 , grade 4 to 3 etc, with that of total recovery of neglect from grade 5 to 0 over 3 months are derived as follows:

$$
D^{1}=\frac{100-X^{1}}{\frac{10^{10}-X^{1} \cdot X^{2} \cdot X^{3} \cdot X^{4} \cdot X^{5}}{10^{8}}}=
$$$$
12 \cdot 5 / 41 \cdot 69=0 \cdot 30
$$

$$
D^{2}=\frac{100-X^{2}}{\frac{10^{10}-X^{1} \cdot X^{2} \cdot X^{3} \cdot X^{4} \cdot X^{5}}{10^{8}}}=
$$

$$
11 \cdot 12 / 41 \cdot 66=0 \cdot 27
$$

$D^{3}=$ etc

The relative value of stage 1 of recovery, that is, recovery from grade 5 to 4 , expressed as a percentage is given by the relative difficulty of recovery from grade 5 to 4 divided by the total difficulty of recovery from grade 5 to 0 , that is,

$$
\frac{D^{1}}{D^{1}+D^{2}+D^{3}+D^{4}+D^{5}} \times 100 \%=
$$


The relative value of stage 2 of recoveryrecovery from grade 5 to 3 is given by the relative difficulty of recovery from grade 5 to 4 plus that of recovery from grade 4 to 3 divided by the total difficulty of recovery from grade 5 to 0 that is

$$
\begin{array}{r}
\frac{D^{1}+D^{2}}{D^{1}+D^{2}+D^{3}+D^{4}+D^{5}} \times 100 \%= \\
0.5669 / 1 \cdot 2236=46 \%
\end{array}
$$

The relative values of stage 3 of recovery (to grade 2), stage 4 of recovery (to grade 1) and stage 5 of recovery (to grade 0 ) can be similarly deduced.

By definition, stage 0 , that of no recovery from grade 5 , is equal to $0 \%$. For reading the menu, grade 4 neglect therefore represented $25 \%$ of total recovery; grade 3 neglect represented $46 \%$ recovery; grade $2,67 \%$; grade 1 , $80 \%$; and grade $0,100 \%$ recovery. 